\title{
Optimizing Alzheimer's Disease Therapy Using a Neural Intelligent Agent-Based Platform
}

\author{
Hamdi Ben Abdessalem, Alexie Byrns, Claude Frasson \\ Département d'Informatique et de Recherche Opérationnelle, Université de Montréal, Montréal, Canada \\ Email: hamdi.ben.abdessalem@umontreal.ca, alexie.byrns@umontreal.ca, frasson@iro.umontreal.ca
}

How to cite this paper: Abdessalem, H.B., Byrns, A. and Frasson, C. (2021) Optimizing Alzheimer's Disease Therapy Using a Neural Intelligent Agent-Based Platform. International Journal of Intelligence Science, 11, 70-96.

https://doi.org/10.4236/ijis.2021.112006

Received: February 23, 2021

Accepted: April 5, 2021

Published: April 8, 2021

Copyright $\odot 2021$ by author(s) and Scientific Research Publishing Inc. This work is licensed under the Creative Commons Attribution International License (CC BY 4.0).

http://creativecommons.org/licenses/by/4.0/

cc) (i) Open Access

\begin{abstract}
Alzheimer's disease affects millions of persons every year. Negative emotions such as stress and frustration have a negative impact on memory function and Alzheimer's patients experience more negative emotions than healthy adults. Non-pharmacological treatment such as immersion in virtual environments could help Alzheimer patients by reducing their negative emotions, but it has restrictions and requirements. In this work, we present three virtual reality relaxing systems in which the patients are immersed in relaxing environments. We propose to use intelligent agents in order to adapt the relaxing environment to each participant and optimize its relaxation effect. The intelligent agents track the emotions of patients using electroencephalography as input in order to adapt the environments. We designed each system with different levels of intelligence in order to analyze the impact of the adaptation on the patients. Experiments were performed for each system on participants with subjective cognitive decline. Results show that these relaxing systems can reduce negative emotions and improve participants' memory performance. The positive effects on affective state and memory persisted for a longer period of time and were generally more effective for the systems with more intelligence. We believe that the combination of a relaxing environment, virtual reality, intelligent agents for adapting the environment, and brain assessment is a promising method for helping Alzheimer's patients.
\end{abstract}

\section{Keywords}

EEG, Brain Assessment, Intelligent Agent, Virtual Reality, Alzheimer's Disease, Subjective Cognitive Decline, Emotions, Adaptation, Emotional Intelligence

\section{Introduction}

Alzheimer's disease (AD) is known for its devastating effects on memory and 
other cognitive functions, but the emotional and psychological aspects of the disease are often overlooked. Anxiety, apathy and dysphoria are common symptoms of the disease that can significantly reduce wellbeing [1].

Individuals aged 65 and older are the most vulnerable to the disease, with vulnerability increasing with age [2]. Early signs of the disease may include such things as loss of smell [3], disorientation [4] and an increased frequency in memory lapses [5]. $\mathrm{AD}$ is a neurodegenerative disease and therefore progressively gets worse over time. At its earliest phase, it is often characterized by a state of Subjective Cognitive Decline (SCD). With the progression of the disease, a large proportion of $\mathrm{AD}$ patients experience symptoms of depression and increased levels of anxiety.

Some pharmacological interventions may reduce symptoms of $\mathrm{AD}$, but none can cure the disease. AD causes negative emotions such as apathy and frustration to settle in patients renders them emotionally unresponsive [6]. New forms of non-pharmacological treatments aiming to alleviate patients are in great demand; especially relaxing techniques that help reduce negative emotions. Some researchers showed that non-pharmacological interventions, including music therapy and psychotherapy, can have positive effects on cognitive function, behavioral problems, mood and functional abilities for older adults suffering from mild cognitive impairment or dementia [7]. Bearing these benefits in mind, this study focuses on relaxing Virtual Reality (VR) environments as a type of nonpharmacological treatment.

VR environments have the advantage that they can be designed, controlled and configured more easily in comparison with real environments. VR has the potential of creating a therapeutic environment isolated from external disturbances offering complete control of the environment to the experimenters.

Furthermore, techniques such as electroencephalography (EEG) and eye tracking have been used to assess the emotional state of the human being [8] [9]. These techniques help track AD patients' negative emotions. By using EEG, we aim to get an inside view of our participants' emotion. Combined with EEG, intelligent agents can intervene on relaxing VR environments in order to adapt them for each user. The EEG inputs allow the agents to understand patients' emotional reactions so they can change environment parameters according to them.

We conducted experiments with SCD participants in order to verify the following hypotheses: H1: Relaxing environments reduce negative emotions, H2: Relaxing environments improve attention and memory performance and H3: Intelligent Agents can adapt relaxing environments in order to optimize its effect.

The rest of this paper is organized as follows. In Section 2, we give an overview of the related works. Section 3 presents our approach. In Sections 4, 5 and 6 we present the three relaxing systems we developed. Section 7 details the experimental procedure, the cognitive tests, and the physiological sensors that we use. Finally, in Section 8 we present and discuss the obtained results. 


\section{Related Works}

\subsection{Relaxation Environment as Treatment for Alzheimer's Disease}

\section{Travel Theory}

Travel therapy is not a particularly popular type of intervention, but neither is it particularly explored. Quick research on the topic shows that travel is used for relationship therapy [10] [11], but there is little to no literature on the uses of travel therapy for neurological disorders such as AD other than indoor traveling [12] [13]. We found only one exception; the study carried out by Biamonti and colleagues [14].

In their study, researchers explored the psychological and behavioral impacts that virtual travel could have on $\mathrm{AD}$ patients in two contexts: the first being a mediocre replicate of a physical train, and the second being a more realistic physical train replicate. Results of the study showed that virtual travel by means of a computerized train increased well-being in individuals with AD. More precisely, participants experienced positive changes in wandering, agitation, anxiety, apathy and sleep [14]. These improvements in psychological and behavioral states are valuable, as these are typical symptoms of AD which significantly diminish the quality of life of patients and even caretakers.

We believe that virtual travel influences behavior and psychological states by first influencing emotional states. Indeed, individuals with $\mathrm{AD}$ often require specialized care which generally causes distance between patients and their loved ones. In such situations, feelings of loneliness and isolation are not uncommon. It is well known that loneliness can have negative effects on individuals [15] [16] [17]. Through virtual travel, participants can break the cycle of loneliness and isolation. We believe that virtual travel positively influences emotional states of participants which in turn promote well-being.

\section{Music Theory}

Music as a therapeutic intervention is commonly used in different health conditions, such as autism, brain injury, depression, anxiety and dementia [18] [19] [20] [21]. It has repeatedly shown to be effective in improving psychological and behavioral states.

Effects of music therapy (MT) on psychological state include decreased stress, anxiety and depression, improved emotional states and relationships with caregivers [22] [23] [24] [25]. Studies often report that the participants seem generally happier following the MT intervention, suggesting an increased well-being [26]. Behavioral changes following these interventions include decreased agitation and wandering, two common and potentially dangerous symptoms of the disease. Finally, MT can also be beneficial on a cognitive level. Studies show slowed cognitive decline and improved orientation, memory and other cognitive functions in patients following a MT session [22] [27] [28] [29].

Although the mechanisms by which MT generates positive changes in wellbeing and potential neurological rehabilitation are not clear, its effects are un- 
mistakable. We believe that some of these effects can be attributable to music's capacity to easily influence emotional states. By increasing positive emotions, we believe that participants become in a favorable cognitive environment.

\section{Savannah Theory}

The term savannah refers here to open environments with short grass, deciduous green trees, water, and animal life. Ancient survival challenges led to a correlation between natural landscapes and positive feelings. Savannah as a natural environment brings back the feeling of tranquility and peace [30].

Spreading trees in savannah gives a feeling of security because they provided early humans with a place to observe or hide from predators. The savannah is an ideal place to travel and explore, it attracts attention and relaxes thanks to its varied and pleasant landscapes.

Wilson introduced the world biophilia in 1984, referring to an innate affinity that people have with nature [31]. Savannah landscapes are preferred by modern humans since the first humans lived for thousands of years in the African savannah [32].

In addition, Meidenbauer K. L. et al. [33] demonstrated in their research that adults have aesthetic preferences for natural environments over urban ones which influenced theories like Biophilia to explain why nature is beneficial for mood improvement. They showed that children demonstrated preferences for urban over natural environments and those urban preferences decreased significantly with age. Both adults and children showed cognitive and affective benefits after nature exposure.

We believe that savannah and natural environments are appreciated and help relax $\mathrm{AD}$ patients which will increase positive emotions and decrease negative emotions.

\subsection{Virtual Reality}

The main characteristics of VR are immersion, sense of presence, and interactivity [34]. These characteristics can be very beneficial and thus VR is rapidly gaining interest in many fields. Biocca et al. defined it as a sum of hardware and software systems that attempt to generate a sensory illusion of being present in another environment different from the one where the user is present physically [35].

VR is used as a therapeutic tool in psychology and psychiatry. Dana et al. [36] have used VR to create virtual environments as treatments for certain types of phobias. They created objects, environments and situations simulating exposure therapy which helps patients face their fear. Moreover, Lorenzo G. et al. [37] have used VR systems with children diagnosed with autism spectrum disorders in order to enhance their emotional skills.

VR interventions are also used to treat pain. Tennant M. et al. [38] investigated the treatment efficacy difference between immersive VR and an iPad on oncology patients' physical and emotional states. Participants who had used VR reported greater improvements in their mood states and reductions in their neg- 
ative symptoms. The preliminary results of this study support the use of VR in clinical oncology in order to improve patient well-being.

Moreover, many studies revealed that VR is beneficial with $\mathrm{AD}$ patients. In fact, some studies have indicated that VR intervention could improve cognitive functions of individuals with mild cognitive impairment (MCI) or AD [39] [40].

\subsection{Adaptative Systems and Intelligent Agents}

Adaptative systems are used in many fields to provide personalized experiences for each user. There are many examples of such instances. For instance, in the learning domain, Chaouachi et al. [41] used adaptive systems in order to maintain users in a positive mental state while learning. In the video games domain, Ghali et al. [42] used adaptive systems to improve players' intuitive reasoning in a physics VR game by changing assistance strategies according to players' emotions.

Moreover, many researchers use intelligent agents in order to adapt their systems. For example, Dayong Ye et al. [43] used an agent-based approach in order to adapt their system to alleviate the runtime quality management problem of distributed service-based systems. They claim that multi-agent systems are ideal to model service-based systems and then solve service adaptation problems. Also, Spencer Rugaber et al. [44] used intelligent agents to create a model-based technique for self-adaptation in game-playing agents. Additionally, Ben Abdessalem and Frasson [45] used intelligent agents to adapt video games to players according to their cognitive and emotional states. In their work, they proposed to track and adapt in real-time the parameters of the video game according to players emotions. Similarly, Peña et al. [46] used intelligent agents to adapt their system to learners. The adaptation technique they used is focused on the didactic contents, navigation tools and navigation strategies of students' learning styles. Likewise, Doctor et al. [47] used intelligent agents to monitor dementia patients living in their own homes. Adaptation rules are applied to facilitate short-term tuning of learned behaviors and long-term tracking of behavioral changes.

\subsection{Brain Assessment}

Physiological sensing approaches are used in several studies in the brain assessment field. Electroencephalography (EEG) is one of these approaches which is used to detect and analyze emotional states. Since EEG is non-invasive and inexpensive, it is a simple and effective way to detect, analyze and evaluate emotions and mental states. In fact, researchers can analyze the brain's electrical activity by using EEG in order to understand the brain's inner working. Several works in different fields relied on EEG in the brain assessment [45] [48].

In the field of serious games, Ghali et al. [42] assessed participants' mental states by considering their engagement and frustration in a physics-related game using EEG. They have proposed to improve intuitive reasoning through help strategies based on these two emotional states. 
Furthermore, in the field of learning, studies realized has shown that students' learning skills are improved when they are in a positive mental state. Considering this, Chaouachi et al. [41] developed a system that aims to help students in their learning by keeping them in a positive mental state. The study was based on students' engagement and workload extracted from EEG signals.

In order to assess emotions, some researchers used EEG data. There is a study, in the field of emotions' detection, designed to detect participants' valence (positive or negative emotion) in real-time while they were invited to watch different videos [49].

Considering the related works presented in this section, we propose the use of VR to create relaxing immersive environments as well as the use of Intelligent Agents with EEG as input in order to adapt these environments and optimize their effects to each person.

\section{Our Approach}

Relaxing environments could have positive effects on individuals suffering from $\mathrm{AD}$. In order to analyze and verify this effect, we propose to perform attention and memory tests measuring participants' cognitive performance before and after their immersion in relaxing environments. We propose to analyze AD patients' emotions during the pre-environment tests, the relaxing environments and once more during the post-environment tests. Figure 1 shows the architecture of our approach.

For each environment, we evaluate the participant's cognitive performance before their immersion in the environment (R1) as well as after the immersion (R2). Comparing R1 and R2 enables to assess the system's effect on attention and memory performances.

We propose three VR relaxing systems inspired by the existing theories cited in Section 2 for AD treatments (Virtual Train, Music Therapy and Savannah Theory) with different levels of intelligence: no intelligence for the Virtual Reality Train, some intelligence for the Music Therapy and more intelligence in

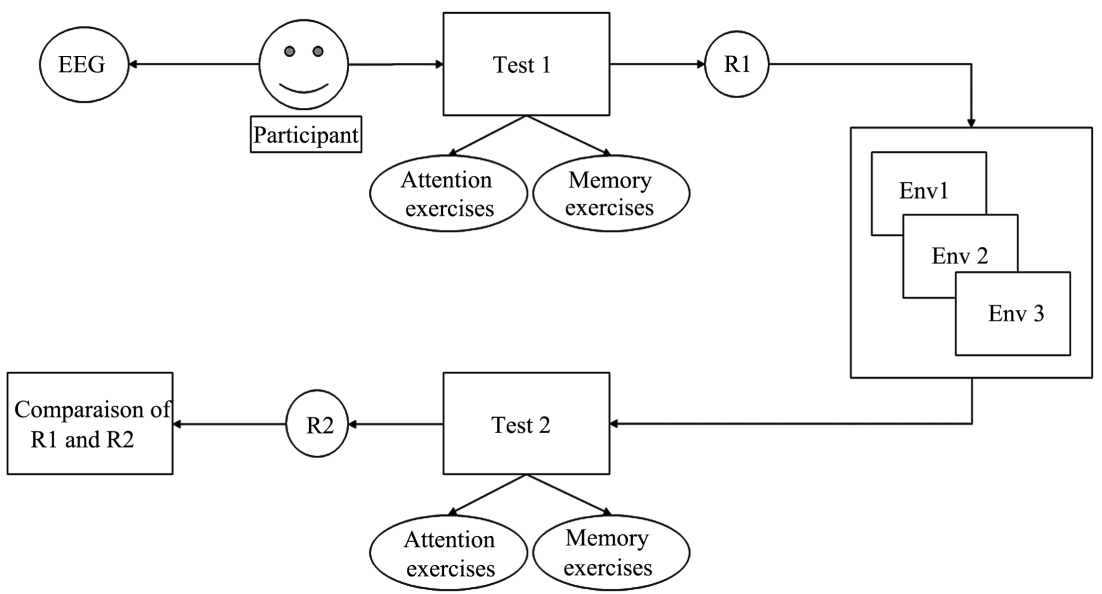

Figure 1. Architecture of our approach. 
Savannah. The following section provides a description of each system. Each environment is created with Unity $3 \mathrm{D}$ game engine which contains a built-in physics engine.

\section{Virtual Reality Train}

The environment described in the Subsection 2.1 was not a virtual environment but a simulation of a real train station. A virtual environment could be a better solution to improve the emotional states of AD patients. The concept of immersion is important for isolating the patient from the real world, providing a sense of presence in the relaxing environment and reducing negative emotions. Thus, we designed and created an immersive VR travel environment. This environment consists of a virtual train in which the participants are immersed, with a family casually sitting next to them (Figure 2).

The virtual train travels through three different natural environments. The first one involves a forest with trees and pacific animals. In the second one, the train travels through a snowy environment with mountains and pacific animals (Figure 3 ). The third and last environment consists of a sunny desert with a warm sun.

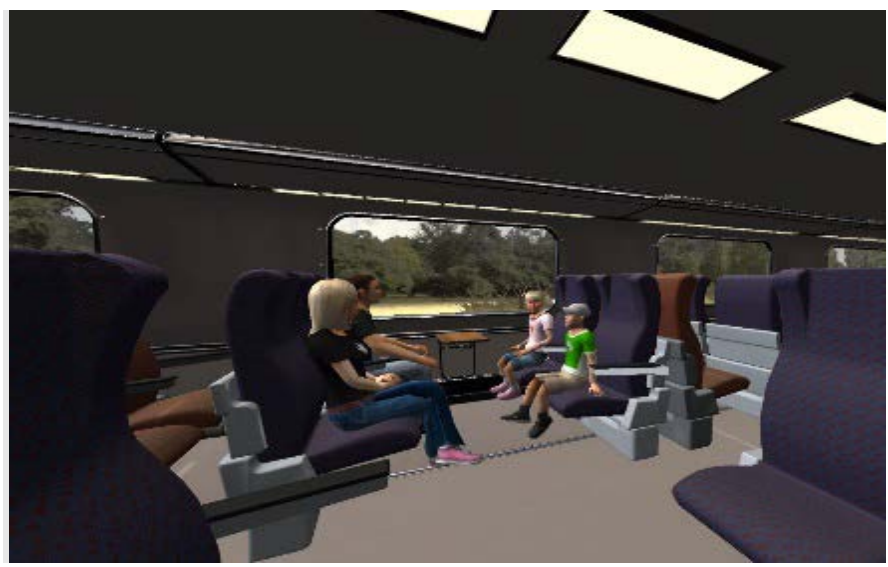

Figure 2. Screen capture of the virtual train.

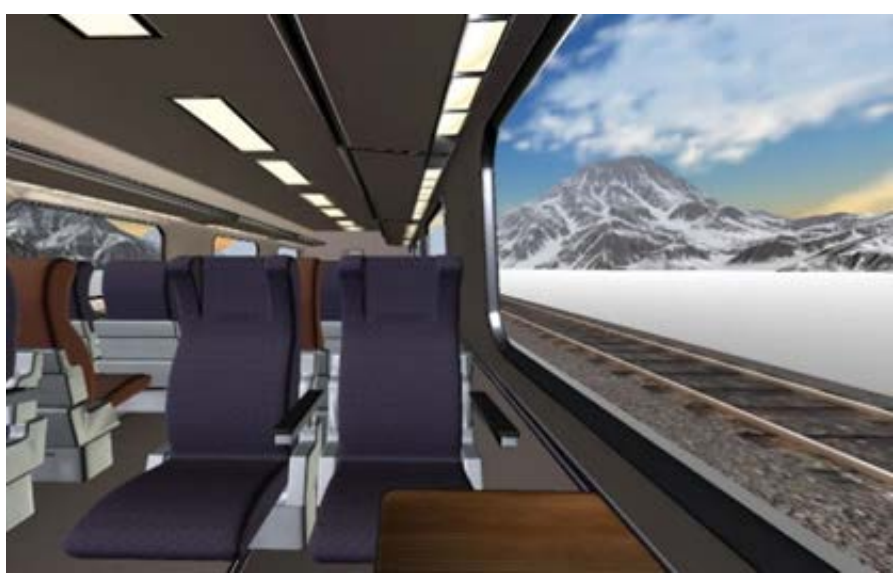

Figure 3. Screen capture of the virtual train (second environment). 


\section{Adaptive Music Therapy}

The Adaptive Music Therapy (AMT) system is composed of a Virtual Music Therapy environment and a Music Adaptation Agent.

\subsection{Virtual Music Therapy}

As mentioned in Section 2.1, Music Therapy (MT) can positively influence psychological states and can help reduce negative emotions. Some studies have shown that VR can be beneficial for individuals suffering from SCD in addition to increasing its positive effect by isolating the participants from external distraction. With this in mind, we designed a Virtual MT therapeutic environment. This environment consists of a music theater in which the patient is immersed, placed in the center and facing the stage up front (see Figure 4).

The environment presents a series of eight songs in the following order: Ave Maria by Franz Schubert, Eine Klein Nachtumusik by Mozart, Clair De Lune by Debussy, La Vie En Rose by Edith Piaf, Everyday by Buddy Holly, La Bamba by Ritchie Valens, and finally What A Wonderful World by Louis Armstrong. These songs were meticulously selected based on music and psychology theories. For example, some songs were chosen due to their structural features; slow tempos and low pitches are generally associated with anxiety reduction [50]. Other songs were selected because they had been popular during years coinciding with the participant's reminiscence bump; the interval between ages 10 and 30 where memories are generally more remembered [51].

A 30 seconds portion of the songs were chosen because they contained melodies with structural features associated with anxiety reduction. Each song is separated from the previous by red curtains which open and close as different songs are presented on stage. For each song, an appropriate selection of instruments is presented on stage, each instrument is animated with the music.

In addition, visual effects are presented above the instruments and the intensity of the light as well as their colors were chosen as a function of the emotional purpose of the song. Scenes were designed with specific shades of red, purple, blue, green and yellow, as they are suggested to be the most pleasant and arousing hues [52]. Figure 4 illustrates two songs with their respective selection of instruments and visual effects.
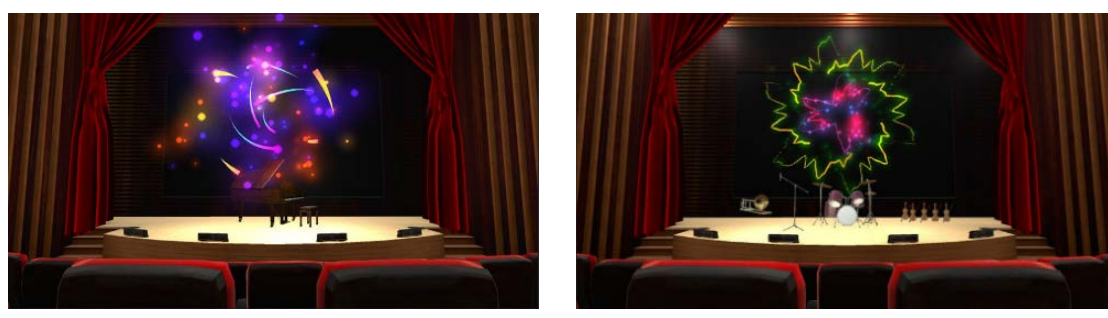

Figure 4. Screen capture of virtual music therapy environment for two different songs.

\subsection{Music Adaptation Agent}

Combining VR with MT could lead to a promising therapy; however, a static en- 
vironment could have different impacts for each participant. Indeed, the intended effect of this environment is to increase emotional wellbeing. Consequently, it is important to adapt the environment to each participant in order to provide the most beneficial therapeutic experience for each individual.

In order to adapt the virtual MT environment to each participant, it is important to track and analyze the emotional impact of each song in real-time. Consequently, we created a Music Adaptation Agent able to record and analyze the users' emotions using Emotiv electroencephalograph (EEG) headset data in real-time. This agent tracks the emotions of the participants while they listen to the eight songs, analyzes the emotional impact of each song while they are playing, and calculates a score for each song according to emotion recordings. Subsequently, based on the scores, the agent detects the song which provokes the best emotional impact and adapts the virtual MT in such a way as to play this song once again for a longer period of time.

\section{Intelligent Savannah Therapy}

The Intelligent Savannah Therapy (IST) system is composed of a Savannah VR environment and a Neural Intelligent Agent.

\subsection{Savannah VR}

As mentioned in Section 2.1, savannahs bring the feeling of tranquility and peace, especially for older adults suffering from SCD, which can help relax and reduce negative emotions. Furthermore, VR in beneficial also for SCD patients and it increases the positive effect of savannah, hence we propose to combine them to create Savannah VR [53].

Savannah VR is a therapeutic environment designed to relax SCD patients. In this environment, participants follow an avatar walking through a savannah. This environment requires only a virtual reality headset to control of the view angle. The dominant colors are warm, the animals are calm and their movement is slow. A soothing piano tune is played in the background at a volume low enough to appreciate the sound of each animal. Figure 5 illustrates screen captures of the environment.

Participants follow a gazelle that moves along a path with breaking points. The gazelle moves slowly to avoid any possible nausea caused by movements within a virtual reality environment. Throughout the experience, the gazelle
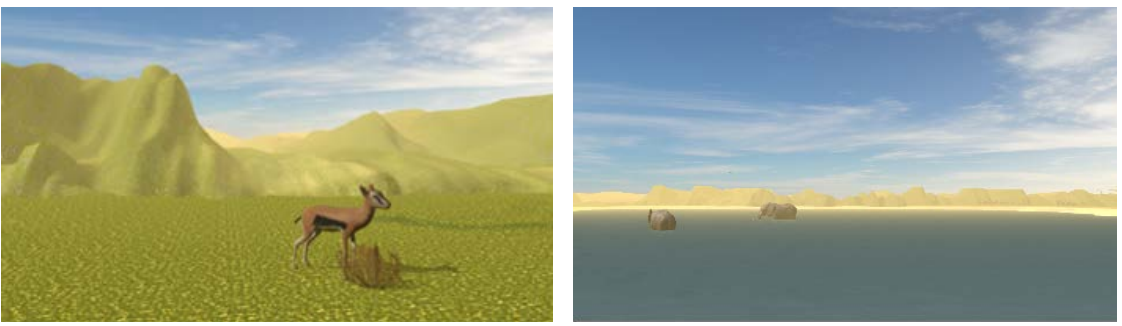

Figure 5. Screenshots of savannah VR. 
remains in front of the participant in such a way as to imitate a third-person view, which is less likely to cause motion sickness [54]. At any moment, participants can look around in 360 degrees by rotating their heads.

Savannah VR could be more beneficial if we made it a dynamic environment which could be changed and adapted to each participant. Integrating an agent capable of adapting the environment could provide an optimized therapeutic experience to each individual.

In order to make the environment adaptable for each participant, we implemented functions that change the environment's parameters in real-time. For instance, one of the adjustable parameters is the color and intensity of the light, since light influences perception and decision-making [55].

\subsection{Neural Agent}

In order to personalize the environment, we propose to use a Neural Agent [45] able to modify the parameters of Savannah VR based on intervention rules triggering a biofeedback approach and more precisely a neurofeedback. Neurofeedback is a type of biofeedback that measures brain waves to produce a signal that can be used as feedback [56].

The Neural Agent consists of an intelligent agent which tracks the emotions of the participants and intervenes on Savannah VR accordingly. This agent has an intervention rules base which possesses multiple intervention rules. Giving the main emotion of the participant, the agent chooses a rule from this rules base and modifies the VR environment to adapt it to the participants. After each intervention, the agent tracks the emotional reactions of the participant to check whether the optimization of participants' emotion was a failure or a success and, if needed, intervenes again on the VR environment.

The Neural Agent is composed of two modules: "Analysis, decision and action" and "Rules base Manager" as well as two databases: "Decisions base" and "Rules base". Figure 6 illustrates the architecture of the Neural Agent and its interactions with the EEG module and Savannah VR. This architecture allows real-time interaction with the user in a neurofeedback loop.

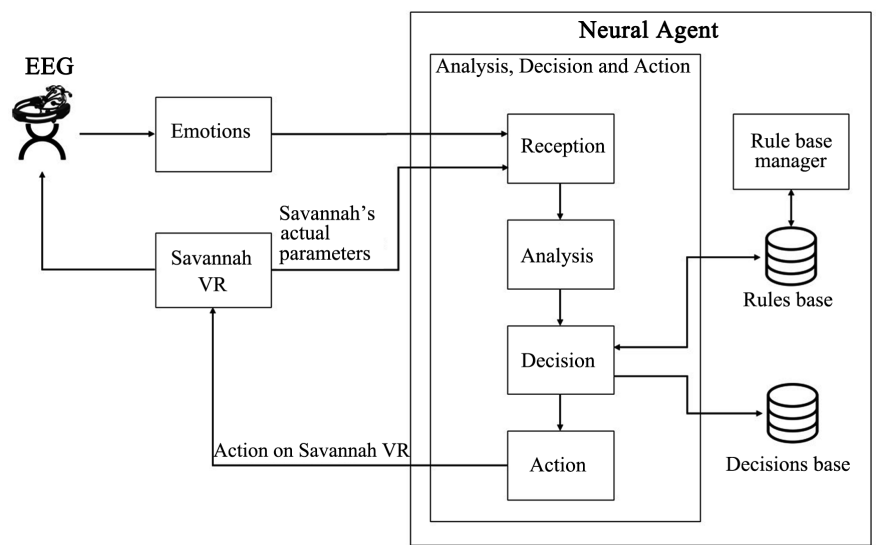

Figure 6. Architecture of the neural agent. 
The Analysis, Decision and Action module has four components which handle reception of data (emotions and information about the status of the environment) and data analysis in order to identify the main emotion and detect whether it corresponds to the expected emotion generated by the environment or not. After that, the module consults the rules base to extract the rule that can be applied and send the action to the environment and stores the decision in the "Decisions base".

After each intervention, the agent observes the emotional reactions of the participant to check whether or not it does modify the emotional state as desired, and updates the weight of the intervention rule accordingly (increase the weight if it worked and decrease it if it doesn't).

The rules base manager module allows for the management of the intervention rules. The three main functionalities are adding, modifying, and deleting of an intervention rule. This module stores the rules in the "Rules base".

\section{Experiments}

We conducted three experiments: one for each system. The details about the participants are the following:

- Virtual Reality Train: 19 participants (11 females) with subjective cognitive decline $(\mathrm{SCD})$ and a mean age $=69.68(\mathrm{SD}=5.49)$;

- Adaptive Music Therapy: 19 participants (13 females) with SCD and a mean age $=72.26(\mathrm{SD}=5.82)$;

- Intelligent Savannah Therapy: 19 participants (12 females) with SCD and a mean age $=71(\mathrm{SD}=8.39)$.

All experiments were conducted at the Institut Universitaire de Geriatrie de Montréal Research Center (CRIUGM) during the day.

All participants attended an eligibility session (lasting one hour) in which we ensured their eligibility. Our eligibility criteria were the following:

- Older than aged 60 of age;

- Francophone;

- Normal or correct-to-normal vision;

- Normal hearing;

- Met the Consortium for the Early Identification of Alzheimer's DiseaseQuebec (CIMA-Q) criteria for SCD:

O Presence of a complaint defined as a positive answer to the following statements: "my memory is not as good as it used to be" "and it worries me";

O MoCA 20 - 30;

O No impairment on the logical memory scale based on the education-adjusted CIMA-Q cut-off scores.

During the eligibility session, participants were provided with oral and written descriptions of the study and invited to sign a consent form. The session then included a series of clinical tests necessary to confirm diagnosis and characterize participants. If the participants were eligible, they were invited to take part in the experiment. 
During the experimental session, participants started by filling pre-session forms. Then we equipped them with an Emotiv Epoc+ EEG headset and invited them to solve the attention and memory exercises described below. Following these exercises, participants were equipped with a FOVE VR headset and one of the relaxing systems presented in the previous sections was launched. Next, participants completed different variants of the same attention and memory exercises. Finally, the experimental session came to an end after filling out the post-session forms. Figure 7 shows the steps of the experimental process.

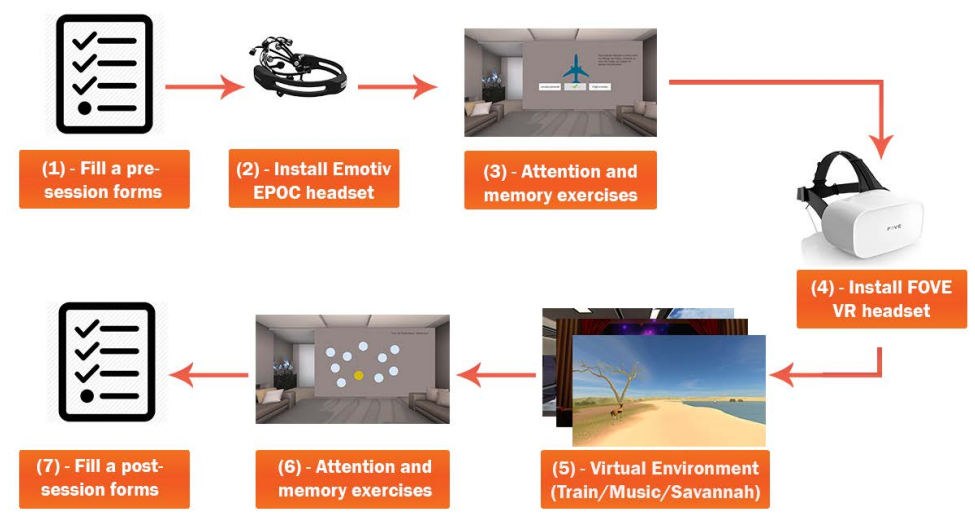

Figure 7. Process of the experiments.

\subsection{Attention Exercises}

\section{Exercise 1.}

In this exercise, we present a series of 5 numbers in auditory form, then a numerical pad is displayed, and the participant is asked to repeat the same sequence; then we hide the pad and present another sound sequence of 3 numbers and the participant is asked to report the sequence in the reverse order.

\section{Exercise 2.}

In the second attention exercise, we present a sound sequence of different letters at a rate of one per second and the participant must click the space bar each time they hear the letter " $A$ ".

\section{Exercise 3.}

The third attention exercise is about naming different objects. For every object, we show an image for 4 seconds, hide it, and then show four letters. Then, the participant is asked to select the first letter of the object's name. Figure 8 illustrates how the participants see an image and then four letters as a suggestion.
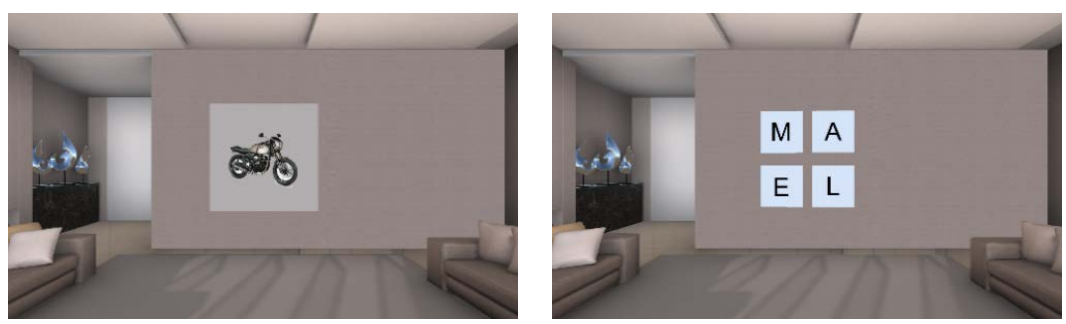

Figure 8. Screenshot of attention exercise 3. 


\subsection{Memory Exercises}

\section{Exercise 4.}

In this exercise the participants are asked to memorize a series of different objects visually or aurally. Then, we present a series of objects and the participants are asked to select whether they saw the object, heard its name, or whether it was never presented. Figure 9 illustrates a case where the participant correctly answered that they saw the image of the dog.

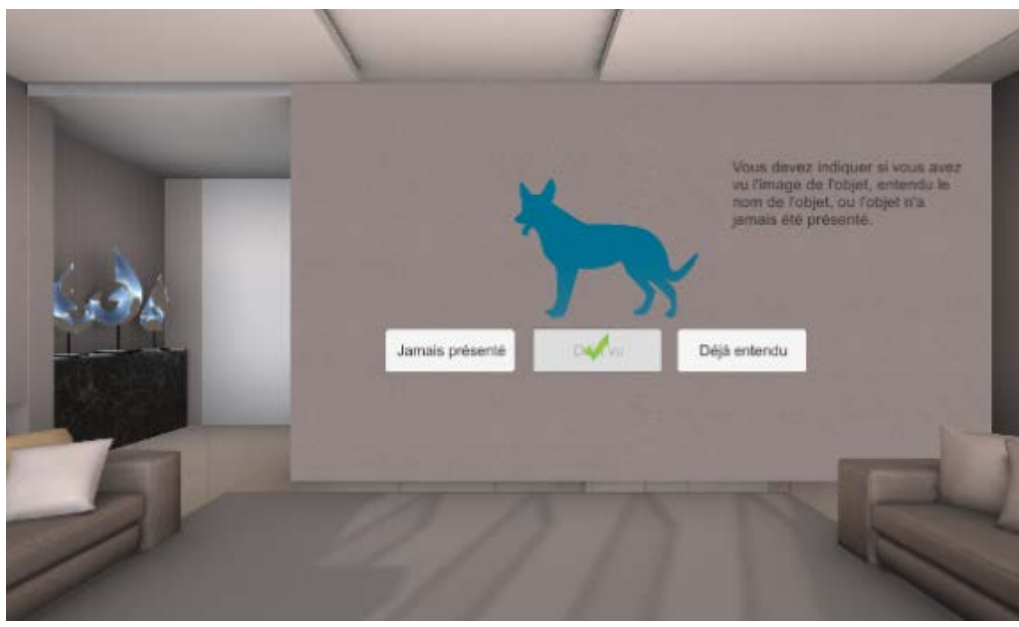

Figure 9. Screenshot of exercise 4.

\section{Exercise 5.}

In the second memory exercise, we present ten circles, and then a series of circles are highlighted one by one in order to create a sequence. The participants are then asked to reproduce the same sequence. We present two sequences with two different levels of difficulty.

\section{Exercise 6.}

In the third and final memory exercise, we present a set of three pictures for a short period of time. Then, we present four sets of three pictures and the participant is asked to identify which set was presented. Figure 10 shows how we present the pictures and a case where the participant chooses the correct answer.
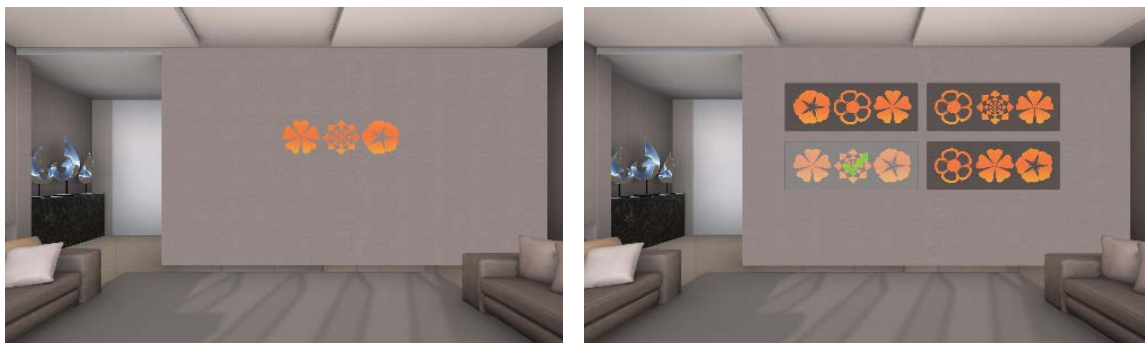

Figure 10. Screenshot of exercise 6.

\subsection{Brain Assessment Using EEG Measures}

In order to track and analyze participants' emotions, we used Emotiv Epoc+ 
EEG headset technology. The headset contains 14 electrodes spatially organized according to the International $10-20$ system, moist with a saline solution. The electrodes are placed at antero-frontal ( $\mathrm{AF} 3, \mathrm{AF} 4, \mathrm{~F} 3, \mathrm{~F} 4, \mathrm{~F} 7, \mathrm{~F} 8)$, fronto-central (FC5, FC6), parietal (P7, P8), temporal (T7, T8) and occipital $(\mathrm{O} 1, \mathrm{O} 2)$ regions with two additional reference sensors placed behind the ears. The detailed position of the measured regions is shown in Figure 11.

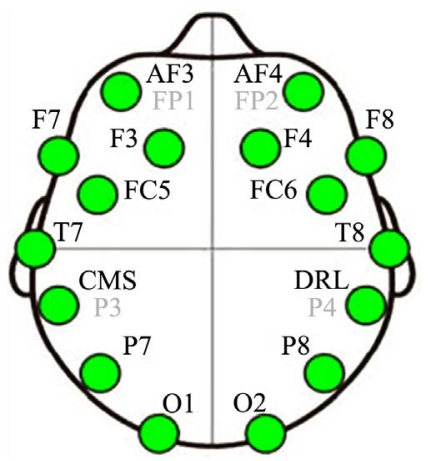

Figure 11. Emotiv headset sensors placement.

The system uses internal algorithms to measure the following mental states: meditation, frustration, engagement, excitement and valence. They were used to assess the effect of the Virtual Reality Train, the Adaptive Music Therapy and Intelligent Savannah Therapy on the emotions of participants and were used as input for the Music Adaptation Agent and The Neural Agent.

Even though we don't have access to Emotiv system proprietary algorithms to infer these mental states, several studies have established the reliability of the output.

\section{Results and Discussion}

\subsection{Therapeutic Train}

In order to verify our first hypothesis that relaxing environments reduce negative emotions, we proceeded by analyzing the participants' negative emotions before and after the VR Train. Results show that the mean frustration of participants before the VR Train was 0.71 (minimum 0.41 and maximum 0.96). The participants' mean frustration during the VR Train was 0.51 (minimum 0.24 and maximum 0.94). After the VR Train, the mean frustration was 0.53 (minimum 0.17 and maximum 0.79). Figure 12 shows a boxplot of the mean frustration before, during and after the travel in the VR Train.

In addition, we conducted a repeated measure ANOVA with participants' frustration level as dependent variable. Results revealed a significant decrease of the frustration during the VR Train and the effect was still observed after the VR Train immersion $(\mathrm{p}=0.0011$ and $\mathrm{F}=7.7218)$. Table 1 details the ANOVA results and shows that the mean frustration level decreased from 0.705 to 0.513 and kept the positive effect after the VR train by increasing only to 0.534 . 


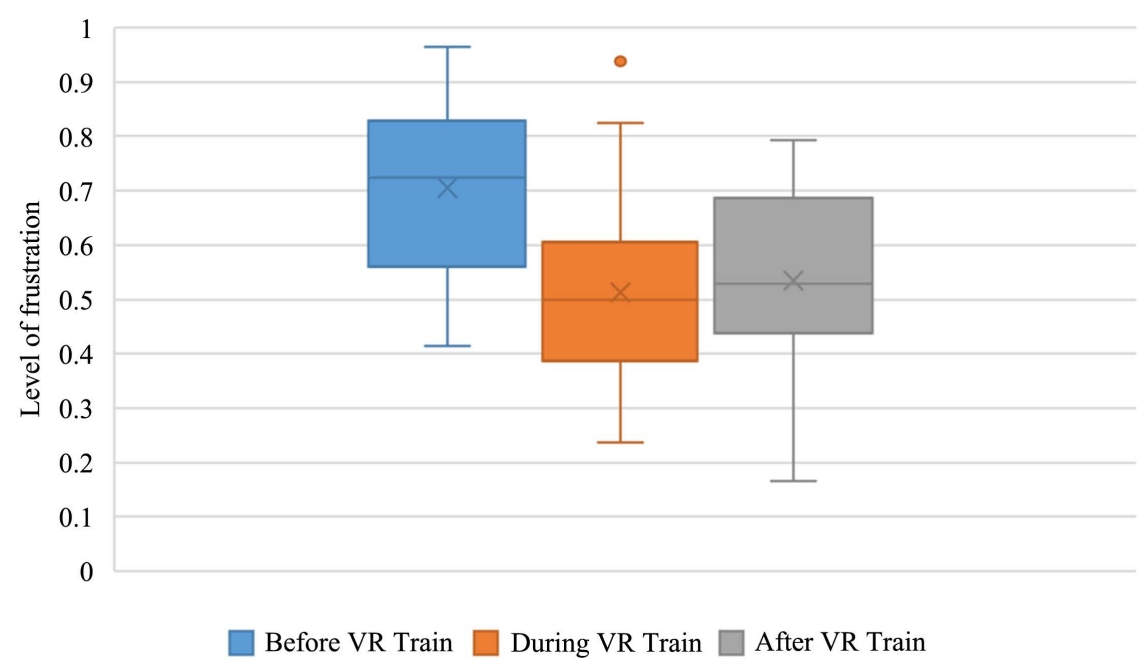

Figure 12 Participants' level of frustration before, during and after VR train.

Table 1. ANOVA mean frustration (VR Train).

\begin{tabular}{cccc}
\hline & Before VR Train & During VR Train & After VR Train \\
\hline Mean & 0.705 & 0.513 & 0.534 \\
SD & 0.148 & 0.186 & 0.158 \\
N & & 19 & \\
F & 7.7218 & \\
P & 0.0011 & \\
\hline
\end{tabular}

Thus, the frustration decreased when the participants were in the VR Train and the positive effect on frustration was preserved following the immersion.

In order to verify our second hypothesis which is: relaxing environments improve attention and memory performance, we started by comparing the performance improvement of the attention and memory exercises by calculating the average mean performance of all participants before and after the VR train. Figure 13 shows the obtained results in which we can notice a clear difference between the improvement of performance on the attention exercises versus the memory exercises.

These results show a clear increase in memory performance following the VR Train and in some cases an improvement in attention abilities.

In order to have more detailed results, we also compared the participants' performances before and after the VR train for each exercise. The mean improvement results of each exercise are shown in Table 2.

The biggest performance improvement is observed in exercise 5 and corresponds to a mean $20 \%$ improvement. In addition, we performed a paired t-test statistical test with participants' scores for exercise 5 before and after the VR Train as parameters. The obtained result is $t=2.293$ and $p=0.017$, this result is significant at $\mathrm{p}<0.05$. 


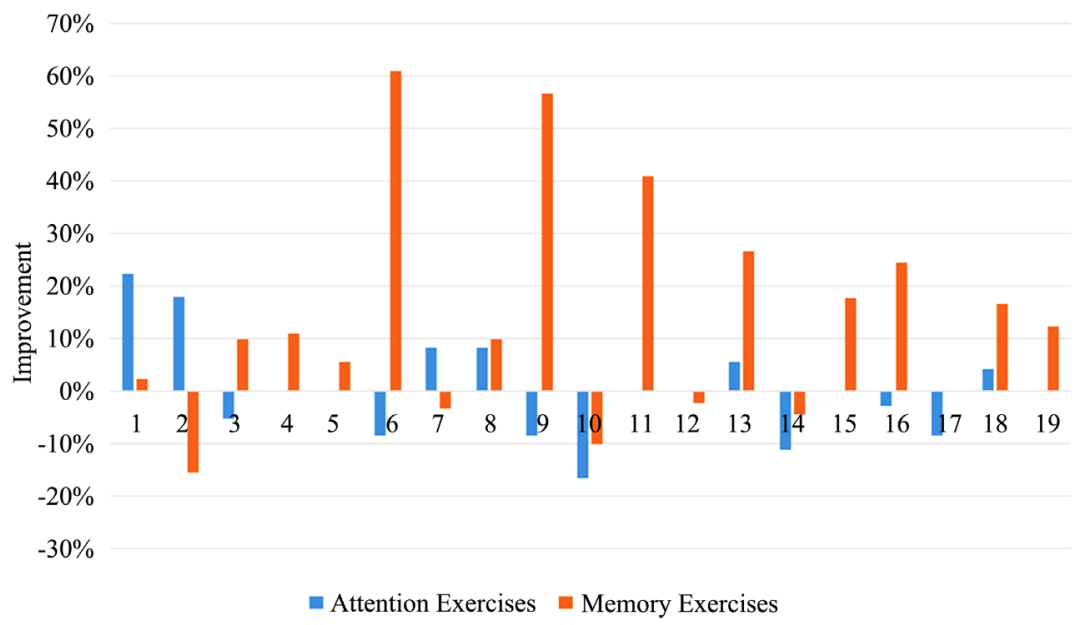

Figure 13. Performance improvement for attention and memory exercises (VR Train).

Table 2. Mean performance improvement (VR train).

\begin{tabular}{ccccccc}
\hline & Exercise 1 & Exercise 2 & Exercise 3 & Exercise 4 & Exercise 5 & Exercise 6 \\
\hline Mean Improvement & $-6.58 \%$ & $0.48 \%$ & $7.02 \%$ & $10.53 \%$ & $\mathbf{2 0 \%}$ & $10.53 \%$ \\
\hline
\end{tabular}

\subsection{Adaptive Music Therapy}

For the second system, we also analyzed the negative emotions before and after Adaptive Music Therapy. We analyzed the level of frustration of the participants and results show that the mean frustration before AMT was 0.691 (minimum 0.377 and maximum 1.0). Their level of frustration during AMT was 0.457 (minimum 0.213 and maximum 0.824). After AMT, their level of frustration was 0.517 (minimum 0.238 and maximum 0.927). Figure 14 shows a boxplot of the mean frustration before, during and after the Adaptive Music Therapy immersion.

In addition, we conducted a repeated measure ANOVA with participants' frustration level as dependent variable. Results revealed a decrease of the frustration during AMT and the effect was still observed after the immersion ( $\mathrm{p}=0.0011$ and $\mathrm{F}=8.26316$ ). Table 3 details the ANOVA results and shows that the mean frustration level decreased from 0.691 to 0.457 , and that the positive effect was kept after AMT and increased only to 0.517.

In order to verify our second hypothesis for the second system, we compared the improvement of attention and memory exercises by calculating the average mean performance of all participants before and after AMT. Figure 15 shows the obtained results.

These results show a difference between the improvements of performance on the attention exercises versus the memory exercises. We notice a clear increase of memory performance after AMT for most participants and an increase in attention performances for nearly half the participants.

We also compared the performance of the participants before and after AMT for each exercise. The mean improvement results of each exercise are shown in Table 4. 


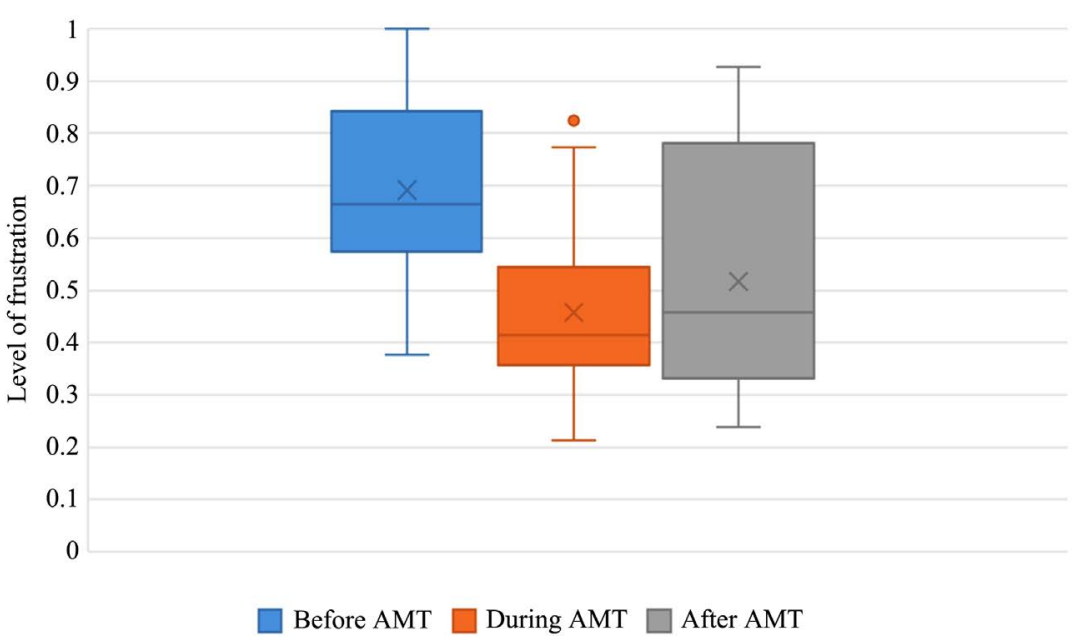

Figure 14. Participants' level of frustration before, during and after AMT.

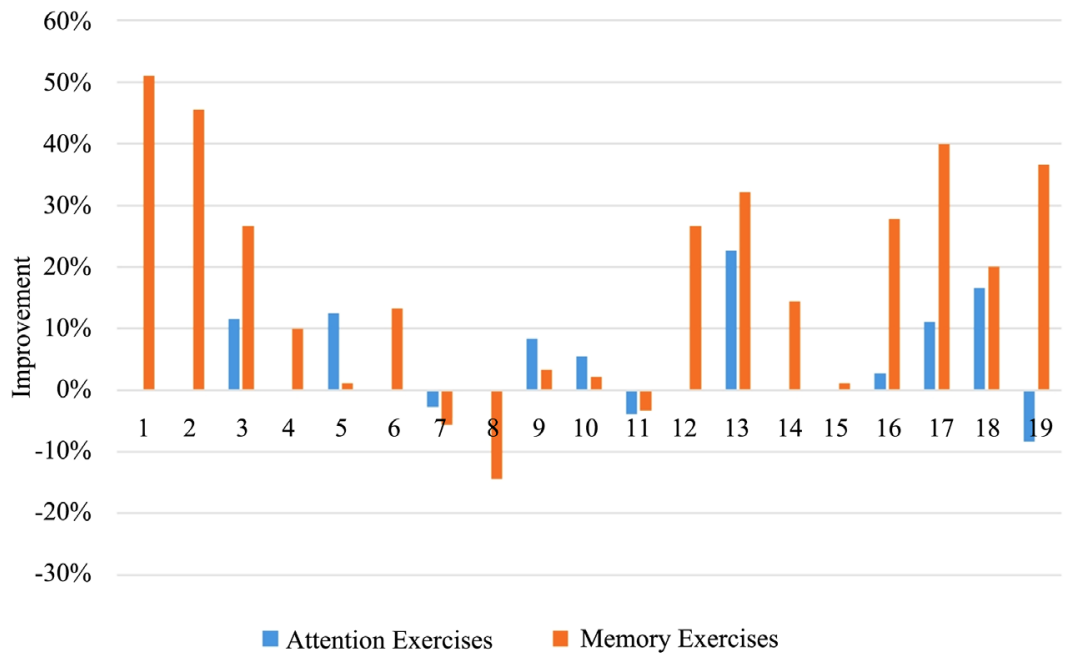

Figure 15. Performance improvement for attention and memory exercises (AMT).

Table 3. ANOVA Mean Frustration (AMT).

\begin{tabular}{cccc}
\hline & Before VR Train & During VR Train & After VR Train \\
\hline Mean & 0.691 & 0.457 & 0.517 \\
SD & 0.184 & 0.150 & 0.212 \\
N & & 19 & \\
F & & 8.2631 & \\
P & & 0.0011 & \\
\hline
\end{tabular}

Table 4. Mean performance improvement (AMT).

\begin{tabular}{ccccccc}
\hline & Exercise 1 & Exercise 2 & Exercise 3 & Exercise 4 & Exercise 5 & Exercise 6 \\
\hline Mean Improvement & $6.58 \%$ & $1.91 \%$ & $3.51 \%$ & $6.14 \%$ & $8.95 \%$ & $\mathbf{3 6 . 8 4 \%}$ \\
\hline
\end{tabular}

The most noticeable improvement is observed in exercise 6, with a mean improvement of $36.84 \%$. In addition, we performed a paired t-test statistical test with participants' scores for exercise 6 before and after the AMT as parameters. 
The obtained result is $\mathrm{t}=3.440$ and $\mathrm{p}=0.0029$, this result is significant at $\mathrm{p}<$ 0.05 .

As we mentioned is Section 5.2, the Music Adaptation Agent intelligently adapts the environment by choosing the most efficient song as a function of the participant's emotional response. Therefore, we analyzed the mean frustration during AMT before and after the agent's adaptation. Results showed that the general mean frustration for all participants, before the agent's adaptation, was 0.48 (minimum 0.29 and maximum 0.84 ) and dropped to 0.41 (minimum 0.18 and maximum 0.77 ) after the agent's adaptation. Individual results are shown in Figure 16.

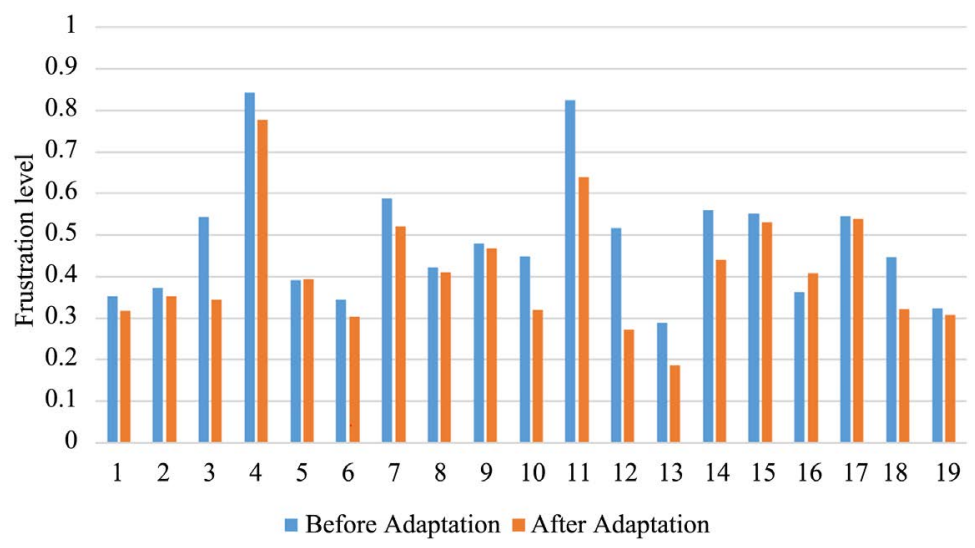

Figure 16. Histogram of mean frustration level before and after agent's adaptation in AMT.

We can observe a frustration decrease after the adaptation for most participants, except for two; only participants 5 and 16 failed to show the effect.

In order to further analyze and support these results, we conducted a t-test for 2 dependent means where the parameters were the mean frustration before and after the adaptation. The obtained results are $t=-2.2082$ and $p=0.040$. This result is significant at $\mathrm{p}<0.05$.

These results support our third hypothesis which is: intelligent agents can adapt relaxing environments in order to optimize its effect, and shows that it is possible to intelligently optimize the environment in order to reduce negative emotions.

\subsection{Intelligent Savanah Therapy}

In addition to the results obtained in the previous section, and in order to support our first hypothesis, we analyzed the negative emotions before and after the Intelligent Savannah Therapy. We analyzed the participants' level of frustration and results show that the mean frustration of participants before IST was 0.68 , (minimum 0.24 and maximum 0.98). The participants' mean frustration during IST was 0.57 (minimum 0.31 and maximum 0.88 ). After IST, the mean frustration was 0.55 (minimum 0.28 and maximum 0.91 ). Figure 17 shows a boxplot of the mean frustration before, during and after IST. 


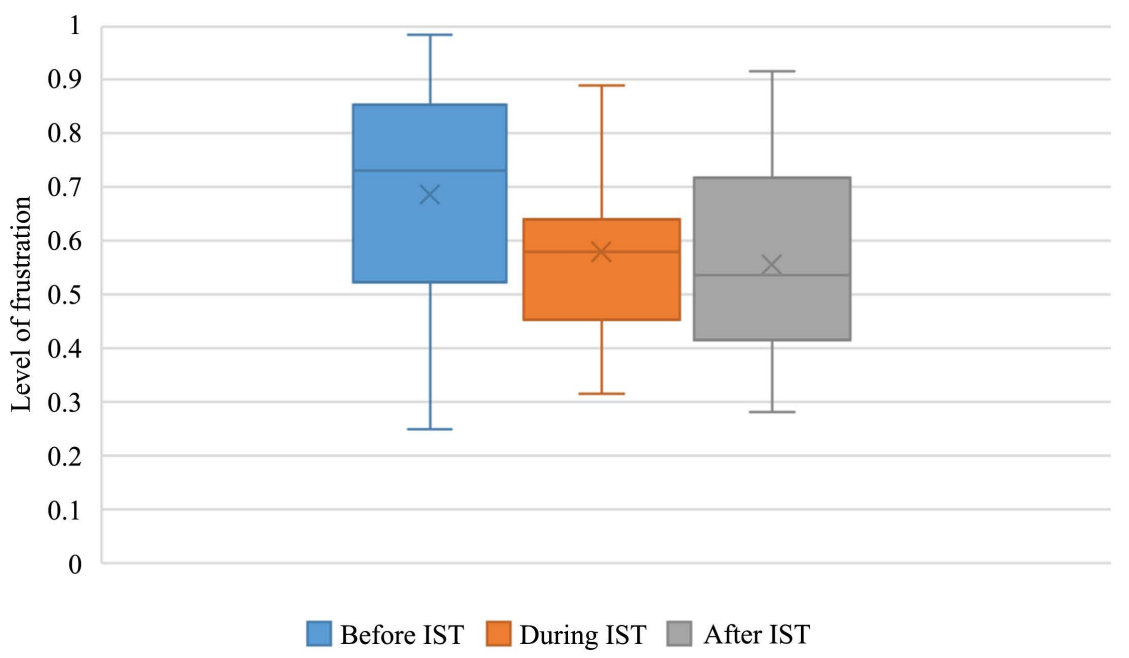

Figure 17. Participants' level of frustration before, during and after IST.

In addition, we conducted a repeated measure ANOVA with participants' frustration level as dependent variable. Results revealed a decrease of the frustration during IST and the effect was still observed after the immersion ( $\mathrm{p}=0.0345$ and $\mathrm{F}=3.7018$ ). Table 5 details the ANOVA results and shows that the mean frustration level decreased from 0.685 to 0.579 and further decreased after IST to 0.555 .

In order to support the results obtained in Sections 8.1 and 8.2 for our second hypothesis, we compared the improvement of attention and memory exercises by calculating the average mean performance of all participants before and after IST. Figure 18 shows the obtained results.

These results show a clear increase of memory performance after IST for most participants (17) and an increase in attention performance for almost half the participants (8).

We also compared the performance of the participants before and after IST for each exercise. The mean improvement results of each exercise are shown in Table 6.

The largest improvement is observed in exercise 6 by $34.21 \%$. In addition, we performed a paired t-test statistical test with participants score of exercise 6 before and after the IST as parameters. The obtained result is $t=3.9804$ and $p=$ 0.0008 . This result is significant at $\mathrm{p}<0.05$.

The performance improvement for exercise 5 was also considerable, with a mean of $16.84 \%$. This led us to perform another paired t-test with participants score of exercise 5 before and after the IST as parameters. The obtained result is $t$ $=2.9682$ and $\mathrm{p}=0.0082$, which is significant at $\mathrm{p}<0.05$.

As mentioned, in Section 6.2, the Neural Agent adapts the environment to personalize its effect to each participant. Thus, in order to confirm our third hypothesis and support the results found in Section 8.2, we analyzed the mean frustration level of each participant before and after the agent's adaptation and personalization of the environment. The Neural Agent intervenes in the 


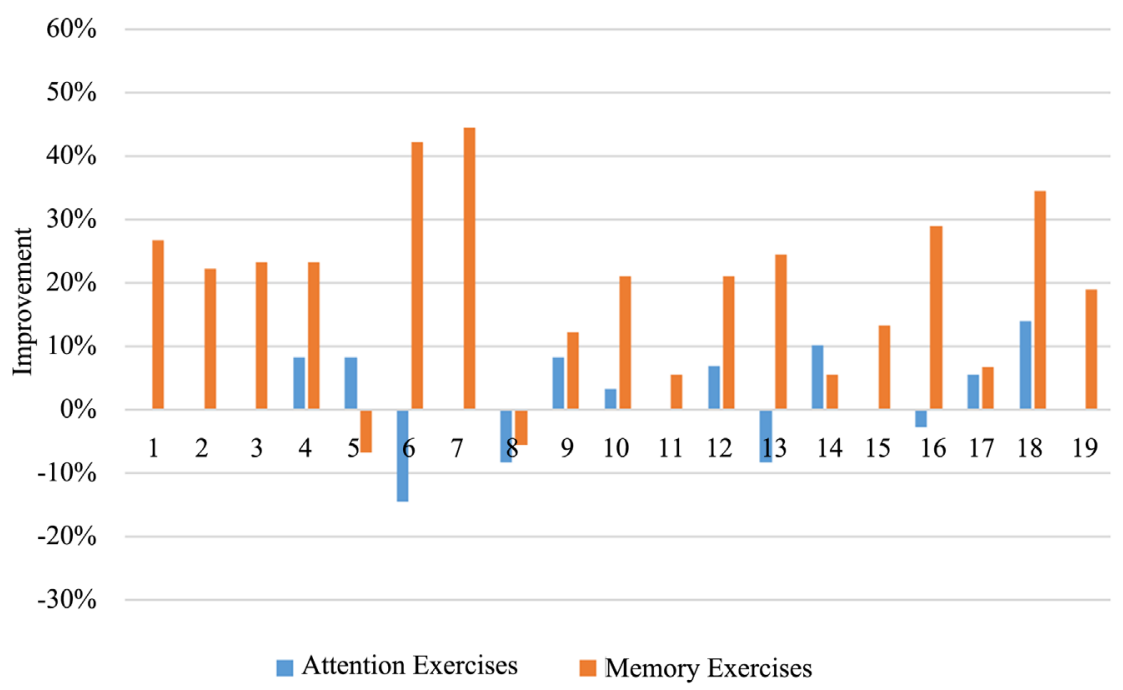

Figure 18. Performance improvement for attention and memory exercises (IST).

Table 5. ANOVA mean frustration (IST).

\begin{tabular}{cccc}
\hline & Before IST & During IST & After IST \\
\hline Mean & 0.685 & 0.579 & 0.555 \\
SD & 0.210 & 0.162 & 0.176 \\
N & & 19 & \\
F & 3.7018 & \\
P & 0.0345 & \\
\hline
\end{tabular}

Table 6. Mean performance improvement (IST).

\begin{tabular}{ccccccc}
\hline & Exercise 1 & Exercise 2 & Exercise 3 & Exercise 4 & Exercise 5 & Exercise 6 \\
\hline Mean Improvement & $-1.32 \%$ & $0.96 \%$ & $5.26 \%$ & $6.14 \%$ & $16.84 \%$ & $\mathbf{3 4 . 2 1 \%}$ \\
\hline
\end{tabular}

environment and then checks whether its changes modify the participant's emotion in the expected way. We analyzed the mean frustration before and after each time the agent intervened and mark the intervention as a success.

Results show that the mean frustration for all participants before the agent's successful interventions was 0.57 (minimum 0.31 and maximum 0.88 ) and dropped to 0.53 (minimum 0.28 and maximum 0.81 ) after the agent's adaptation. Individual results are shown in Figure 19.

We can observe a frustration decrease after the adaptation for most participants except for two. Only participants 5 and 8 failed to show the effect.

In order to support these results, we conducted a t-test for 2 dependent means where the parameters were the mean frustration before and after the agent's intervention. The obtained results are $t=-4.5815$ and $p=0.0002$, which is significant at $\mathrm{p}<0.05$. These results confirm that we can intelligently adapt the relaxing environment in order to optimize its effect. 


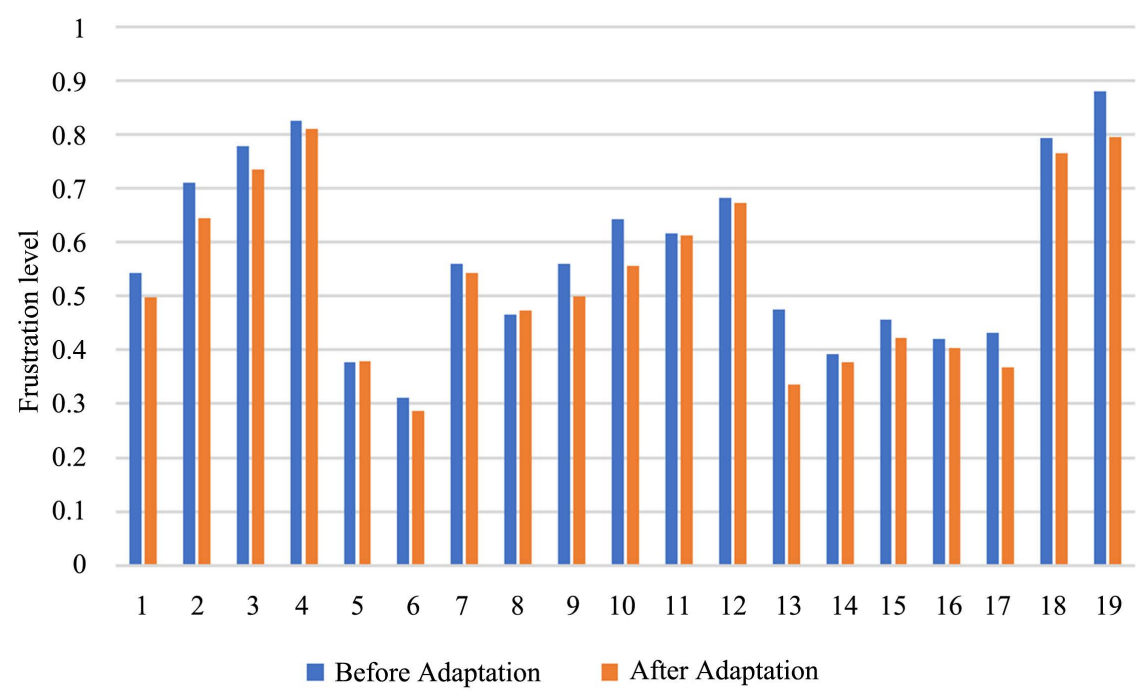

Figure 19. Histogram of mean frustration level before and after agent's adaptation in IST.

\subsection{Overall Results}

The results presented in the previous subsection support our first hypothesis and show that our three relaxing systems successfully reduce participants' frustration. This effect varies with the level of intelligence of the system. In Table 7, we present the participants' mean frustration before, during and after the three relaxing systems. We note that for our three systems, the frustration drops during all systems, but the effect of relaxation stays longer with more adaptation and more intelligence. The system with the Neural Agent is the only one which makes the effect of relaxation persist longer even after the immersion and does not exceed the initial level of frustration. These results support our third hypothesis and show that intelligent agents can optimize the effect of relaxing systems.

Table 7. Comparison of mean frustration.

\begin{tabular}{cccc}
\hline & Before & During & After \\
\hline VR Train & 0.705 & 0.513 & 0.534 \\
AMT & 0.691 & 0.457 & 0.517 \\
IST & 0.685 & $\mathbf{0 . 5 7 9}$ & $\mathbf{0 . 5 5 5}$ \\
\hline
\end{tabular}

In addition, the results presented in the previous subsection, support our second hypothesis, and show that our three systems improve participants' memory performance and in some cases attention performance too. In Table 8, we present a comparison of the mean performance improvement for memory exercises. We note that for our three systems, the memory performance increases, but the level of improvement is more important when the system is more intelligent. The largest mean improvement is observed in the system with the Neural Agent. This therefore supports our third hypothesis shows that intelligent agents can optimize the effect of our therapeutic systems. 
Table 8. Comparison of mean memory performance improvement.

\begin{tabular}{ccccc}
\hline & Exercise 4 & Exercise 5 & Exercise 6 & Mean \\
\hline VR Train & $10.53 \%$ & $20 \%$ & $10.53 \%$ & $13.69 \%$ \\
AMT & $6.14 \%$ & $8.95 \%$ & $36,84 \%$ & $17.31 \%$ \\
IST & $6.14 \%$ & $16.84 \%$ & $34.21 \%$ & $19.06 \%$ \\
\hline
\end{tabular}

\subsection{Work Limitations}

The results presented in the previous section show that our approach has a great potential to reduce negative emotions and improve memory performances. However, our work has some limitations; First, we could not perform experiments on more than 19 participants per system. Future lab research will require additional experiments in order to verify the effect on our environments is a larger scale and consequently confirm the obtained effect. Second, our approach requires the use of two headsets (EEG and VR headsets) in order to collect experiments' data and some participants were not very comfortable using both headsets for a long period of time. This could explain the decrease of performance of some participants.

In terms of the environments themselves, each participant had a different preference. Although most of the participants appreciated the environments, which led to a general positive effect detailed in the previous section, their effects were not unanimous, as some participants did not benefit from the environment's therapeutic effect as much as others. For example, in Train VR system, some participants experienced motion sickness problems. For AMT, some songs could have triggered the recollection of negative memories, ultimately negatively influencing their emotional state. Finally, for IST, some participants did not enjoy or were uncomfortable in the presence of animals. By influencing emotional states, all of these factors could have contributed to a decrease in the participants' performance.

In addition, we also note that this approach would not be feasible for blind individuals since the VR headset is required to the immersive effect. However, two of our environments, namely Train VR and IST, could work for deaf individuals, but the result could be different due to the absence of relaxing background music for Train VR and Volume/Music controlled by the agent in IST.

\section{Conclusions and Future Works}

In this paper, we presented a novel approach that uses Adaptive Intelligent Agents in order to optimize the calming effects of relaxing VR environments on SCD patients. We presented three systems with different levels of intelligent adaptation which aim to relax SCD patients and subsequently improve their memory performance. We performed experiments for each system. Results showed that the three relaxing systems, Virtual Train, Adaptive Music Therapy and Intelligent Savannah Therapy, reduce participants' negative emotions. In addition, these three environments improve memory performances. We note that the 
more intelligence we have in the system, the better the system is at reducing negative emotions and improving memory performances. We conclude that intelligent agents can successfully adapt these relaxing environments in order to optimize the environment's benefit for each participant. As future works, we aim to further push the level of intelligence by creating a personal intelligent agent capable of learning about each participant for more personalized and optimized adaptation effects.

\section{Acknowledgements}

We thank Marc Cuesta, Sylvie Belleville, Caroline Dakoure and Marwa Boukadida for their contributions to this work.

We acknowledge NSERC-CRD (National Science and Engineering Research Council), Prompt and Beam Me Up Labs for funding this work.

\section{Conflicts of Interest}

The authors declare no conflicts of interest regarding the publication of this paper.

\section{References}

[1] Benoit, M., et al. (1999) Behavioral and Psychological Symptoms in Alzheimer's Disease. Dementia and Geriatric Cognitive Disorders, 10, 511-517. https://doi.org/10.1159/000017198

[2] Boyle, P.A., et al. (2019) Attributable Risk of Alzheimer's Dementia Attributed to Age-Related Neuropathologies. Annals of Neurology, 85, 114-124. https://doi.org/10.1002/ana.25380

[3] Mesholam, R.I., Moberg, P.J., Mahr, R.N. and Doty, R.L. (1998) Olfaction in Neurodegenerative Disease: A Meta-Analysis of Olfactory Functioning in Alzheimer's and Parkinson's Diseases. Archives of Neurology, 55, 84-90. https://doi.org/10.1001/archneur.55.1.84

[4] Coughlan, G., Laczó, J., Hort, J., Minihane, A.-M. and Hornberger, M. (2018) Spatial Navigation Deficits-Overlooked Cognitive Marker for Preclinical Alzheimer Disease? Nature Reviews Neurology, 14, 496-506. https://doi.org/10.1038/s41582-018-0031-x

[5] Slot, R.E., et al. (2019) Subjective Cognitive Decline and Rates of Incident Alzheimer's Disease and Non-Alzheimer's Disease Dementia. Alzheimer's \& Dementia, 15, 465-476.

[6] Landes, A.M., Sperry, S.D., Strauss, M.E. and Geldmacher, D.S. (2001) Apathy in Alzheimer's Disease. Journal of the American Geriatrics Society, 49, 1700-1707. https://doi.org/10.1046/j.1532-5415.2001.49282.x

[7] Tsoi, K.K., Chan, J.Y., Ng, Y.-M., Lee, M.M., Kwok, T.C. and Wong, S.Y. (2018) Receptive Music Therapy Is More Effective than Interactive Music Therapy to Relieve Behavioral and Psychological Symptoms of Dementia: A Systematic Review and Meta-Analysis. Journal of the American Medical Directors Association, 19 , 568-576. https://doi.org/10.1016/j.jamda.2017.12.009

[8] Ben Abdessalem, H., Chaouachi, M., Boukadida, M. and Frasson, C. (2019) Toward Real-Time System Adaptation Using Excitement Detection from Eye Tracking. In: 
Coy, A., Hayashi, Y. and Chang, M., Eds., Eds., Intelligent Tutoring Systems, 11528, Springer International Publishing, Berlin, 214-223. https://doi.org/10.1007/978-3-030-22244-4_26

[9] Berka, C., et al. (2007) EEG Correlates of Task Engagement and Mental Workload in Vigilance, Learning, and Memory Tasks. Aviation, Space, and Environmental Medicine, 78, 231-244.

[10] Durko, A.M. and Petrick, J.F. (2016) Travel as Relationship Therapy: Examining the Effect of Vacation Satisfaction Applied to the Investment Model. Journal of Travel Research, 55, 904-918. https://doi.org/10.1177/0047287515592970

[11] Schaler, K. (2009) Travel Therapy: Where Do You Need to Go? Seal Press, Berkeley, California.

[12] Lancioni, G.E., et al. (2013) Technology-Based Orientation Programs to Support Indoor Travel by Persons with Moderate Alzheimer's Disease: Impact Assessment and Social Validation. Research in Developmental Disabilities, 34, 286-293. https://doi.org/10.1016/j.ridd.2012.08.016

[13] Lancioni, G.E., et al. (2013) Supporting Daily Activities and Indoor Travel of Persons with Moderate Alzheimer's Disease through Standard Technology Resources. Research in Developmental Disabilities, 34, 2351-2359. https://doi.org/10.1016/j.ridd.2013.04.020

[14] Biamonti, A., Gramegna, S. and Imamogullari, B. (2014) A Design Experience for the Enhancement of the Quality of Life for People with Alzheimer's Disease. What's On: Cumulus Spring Conference, Averio, 8-10 May 2014, 273-284.

[15] Heinrich, L.M. and Gullone, E. (2006) The Clinical Significance of Loneliness: A Literature Review. Clinical Psychology Review, 26, 695-718. https://doi.org/10.1016/j.cpr.2006.04.002

[16] Cacioppo, J.T. and Cacioppo, S. (2018) The Growing Problem of Loneliness. The Lancet, 391, 426. https://doi.org/10.1016/S0140-6736(18)30142-9

[17] Hawkley, L.C. and Cacioppo, J.T. (2003) Loneliness and Pathways to Disease. Brain, Behavior, and Immunity, 17, 98-105. https://doi.org/10.1016/S0889-1591(02)00073-9

[18] Geretsegger, M., Elefant, C., Mössler, K.A. and Gold, C. (2014) Music Therapy for People with Autism Spectrum Disorder. Cochrane Database of Systematic Reviews, No. 6, CD004381. https://doi.org/10.1002/14651858.CD004381.pub3

[19] Bradt, J., Magee, W.L., Dileo, C., Wheeler, B.L. and McGilloway, E. (2010) Music Therapy for Acquired Brain Injury. Cochrane Database of Systematic Reviews, No. 7, CD006787. https://doi.org/10.1002/14651858.CD006787.pub2

[20] Gutiérrez, E.O.F. and Camarena, V.A.T. (2015) Music Therapy in Generalized Anxiety Disorder. The Arts in Psychotherapy, 44, 19-24. https://doi.org/10.1016/j.aip.2015.02.003

[21] Vink, A.C., Bruinsma, M.S. and Scholten, R.J. (2003) Music Therapy for People with Dementia. Cochrane Database of Systematic Reviews, No. 3, CD003477. https://doi.org/10.1002/14651858.CD003477.pub2

[22] Gallego, M.G. and García, J.G. (2017) Music Therapy and Alzheimer's Disease: Cognitive, Psychological, and Behavioural Effects. Neurología (English Edition), 32, 300-308. https://doi.org/10.1016/j.nrleng.2015.12.001

[23] Ray, K.D. and Mittelman, M.S. (2017) Music Therapy: A Nonpharmacological Approach to the Care of Agitation and Depressive Symptoms for Nursing Home Residents with Dementia. Dementia, 16, 689-710. 
https://doi.org/10.1177/1471301215613779

[24] King, J., et al. (2019) Increased Functional Connectivity after Listening to Favored Music in Adults with Alzheimer Dementia. The Journal of Prevention of Alzheimer's Disease, 6, 56-62. https://doi.org/10.14283/jpad.2018.19

[25] de la Rubia Ortí, J.E., et al. (2018) Does Music Therapy Improve Anxiety and Depression in Alzheimer's Patients? The Journal of Alternative and Complementary Medicine, 24, 33-36. https://doi.org/10.1089/acm.2016.0346

[26] de la Rubia Ortí, J.E., et al. (2019) Music Therapy Decreases Sadness and Increases Happiness in Alzheimer Patients: A Pilot Study. Neuropsychiatry, 8, 739-744. https://doi.org/10.4172/Neuropsychiatry.1000546

[27] Chang, Y., et al. (2015) The Efficacy of Music Therapy for People with Dementia: A Meta-Analysis of Randomised Controlled Trials. Journal of Clinical Nursing, 24, 3425-3440. https://doi.org/10.1111/jocn.12976

[28] Zhang, Y., et al. (2017) Does Music Therapy Enhance Behavioral and Cognitive Function in Elderly Dementia Patients? A Systematic Review and Meta-Analysis. Ageing Research Reviews, 35, 1-11. https://doi.org/10.1016/j.arr.2016.12.003

[29] Peck, K.J., Girard, T.A., Russo, F.A. and Fiocco, A.J. (2016) Music and Memory in Alzheimer's Disease and the Potential Underlying Mechanisms. Journal of Alzheimer's Disease, 51, 949-959. https://doi.org/10.3233/JAD-150998

[30] Frumkin, H. (2001) Beyond Toxicity: Human Health and the Natural Environment. American Journal of Preventive Medicine, 20, 234-240.

https://doi.org/10.1016/S0749-3797(00)00317-2

[31] Wilson, E.O. (1984) Biophilia. Harvard University Press, Cambridge.

[32] Balling, J.D. and Falk, J.H. (1982) Development of Visual Preference for Natural Environments. Environment and Behavior, 14, 5-28.

https://doi.org/10.1177/0013916582141001

[33] Meidenbauer, K.L., et al. (2019) The Gradual Development of the Preference for Natural Environments. Journal of Environmental Psychology, 65, Article ID: 101328. https://doi.org/10.1016/j.jenvp.2019.101328

[34] Walsh, K.R. and Pawlowski, S.D. (2002) Virtual Reality: A Technology in Need of IS Research. Communications of the Association for Information Systems, 8, 296-314. https://doi.org/10.17705/1CAIS.00820

[35] Biocca, F. and Levy, M.R. (1995) Communication in the Age of Virtual Reality.

[36] Horváthová, D. and Siládi, V. (2016) Creating Virtual Environments for Phobia Treatment. Open Computer Science, 6, 138-147. https://doi.org/10.1515/comp-2016-0012

[37] Lorenzo, G., Lledó, A., Pomares, J. and Roig, R. (2016) Design and Application of an Immersive Virtual Reality System to Enhance Emotional Skills for Children with Autism Spectrum Disorders. Computers \& Education, 98, 192-205. https://doi.org/10.1016/j.compedu.2016.03.018

[38] Tennant, M., Youssef, G.J., McGillivray, J., Clark, T.-J., McMillan, L. and McCarthy, M.C. (2020) Exploring the Use of Immersive Virtual Reality to Enhance Psychological Well-Being in Pediatric Oncology: A Pilot Randomized Controlled Trial. European Journal of Oncology Nursing, 48, Article ID: 101804.

https://doi.org/10.1016/j.ejon.2020.101804

[39] Coyle, H., Traynor, V. and Solowij, N. (2015) Computerized and Virtual Reality Cognitive Training for Individuals at High Risk of Cognitive Decline: Systematic Review of the Literature. The American Journal of Geriatric Psychiatry, 23, 335-359. 
https://doi.org/10.1016/j.jagp.2014.04.009

[40] Hill, N.T.M., Mowszowski, L., Naismith, S.L., Chadwick, V.L., Valenzuela, M. and Lampit, A. (2017) Computerized Cognitive Training in Older Adults with Mild Cognitive Impairment or Dementia: A Systematic Review and Meta-Analysis. The American Journal of Psychiatry, 174, 329-340.

https://doi.org/10.1176/appi.ajp.2016.16030360

[41] Chaouachi, M., Jraidi, I. and Frasson, C. (2015) Adapting to Learners' Mental States Using a Physiological Computing Approach. The Twenty-Eighth International Flairs Conference, Hollywood, Florida, USA, 18-20 May 2015, 257-262.

[42] Ramla, G., Hamdi, B.A. and Claude, F. (2017) Improving Intuitive Reasoning through Assistance Strategies in a Virtual Reality Game. The Thirtieth International Florida Artificial Intelligence Research Society Conference, Marco Island, Florida, USA, 22-24 May 2017, 382-387.

[43] Ye, D., He, Q., Wang, Y. and Yang, Y. (2018) An Agent-Based Service Adaptation Approach in Distributed Multi-Tenant Service-Based Systems. Journal of Parallel and Distributed Computing, 122, 11-25. https://doi.org/10.1016/j.jpdc.2018.07.006

[44] Rugaber, S., Goel, A.K. and Martie, L. (2013) GAIA: A CAD Environment for Model-Based Adaptation of Game-Playing Software Agents. Procedia Computer Science, 16, 29-38. https://doi.org/10.1016/j.procs.2013.01.004

[45] Ben Abdessalem, H. and Frasson, C. (2017) Real-Time Brain Assessment for Adaptive Virtual Reality Game : A Neurofeedback Approach. In: Frasson, C. and Kostopoulos, G., Eds., Brain Function Assessment in Learning, 10512, Springer International Publishing, Berlin, 133-143. https://doi.org/10.1007/978-3-319-67615-9_12

[46] Peña, C., Marzo, J.-L. and Rosa, J.D.L. (2002) Intelligent Agents in a Teaching and Learning Environment on the Web.

[47] Doctor, F., Iqbal, R. and Naguib, R.N.G. (2014) A Fuzzy Ambient Intelligent Agents Approach for Monitoring Disease Progression of Dementia Patients. Journal of Ambient Intelligence and Humanized Computing, 5, 147-158. https://doi.org/10.1007/s12652-012-0135-x

[48] Jha, M.K., et al. (2020) Virtual Reality Orientation Game for Alzheimer's Disease Using Real-Time Help System. In: Vlamos, P., Bamidis, P. and Frasson, C., Eds., Brain Function Assessment in Learning, 12462, Springer International Publishing, Berlin, 13-23. https://doi.org/10.1007/978-3-030-60735-7_2

[49] Soleymani, M., Asghari-Esfeden, S., Pantic, M. and Fu, Y. (2014) Continuous Emotion Detection Using EEG Signals and Facial Expressions. Proceedings of the IEEE International Conference on Multimedia and Expo (ICME 2014), Chengdu, 14-18 July 2014, 1-6. https://doi.org/10.1109/ICME.2014.6890301

[50] de la Torre-Luque, A., Caparros-Gonzalez, R.A., Bastard, T., Vico, F.J. and Buela-Casal, G. (2017) Acute Stress Recovery through Listening to Melomics Relaxing Music: A Randomized Controlled Trial. Nordic Journal of Music Therapy, 26, 124-141. https://doi.org/10.1080/08098131.2015.1131186

[51] Valdez, P. and Mehrabian, A. (1994) Effects of Color on Emotions. Journal of Experimental Psychology: General, 123, 394-409. https://doi.org/10.1037/0096-3445.123.4.394

[52] Dakoure, C., et al. (2020) Virtual Savannah: An Effective Therapeutic and Relaxing Treatment for People with Subjective Cognitive Decline. In: Vlamos, P., Bamidis, P. and Frasson, C., Eds., Brain Function Assessment in Learning, 12462, Springer International Publishing, Berlin, 107-112.

https://doi.org/10.1007/978-3-030-60735-7_12 
[53] Monteiro, D., Liang, H.-N., Xu, W., Brucker, M., Nanjappan, V. and Yue, Y. (2018) Evaluating Enjoyment, Presence, and Emulator Sickness in VR Games Based on First- and Third-Person Viewing Perspectives: Effect of Viewing Perspective in VR Gaming. Computer Animation and Virtual Worlds, 29, e1830. https://doi.org/10.1002/cav.1830

[54] Song, S. and Yamada, S. (2019) Ambient Lights Influence Perception and Decision-Making. Frontiers in Psychology, 9, 2685.

https://doi.org/10.3389/fpsyg.2018.02685

[55] Sherlin, L.H., et al. (2011) Neurofeedback and Basic Learning Theory: Implications for Research and Practice. Journal of Neurotherapy, 15, 292-304. https://doi.org/10.1080/10874208.2011.623089

[56] Aspinall, P., Mavros, P., Coyne, R. and Roe, J. (2015) The Urban Brain: Analysing Outdoor Physical Activity with Mobile EEG. British Journal of Sports Medicine, 49, 272-276. https://doi.org/10.1136/bjsports-2012-091877. 